\title{
Crystal structure of diaquabis(5-chlorosalicylaldehydato)cobalt(II), $\mathrm{Co}\left(\mathrm{H}_{2} \mathrm{O}\right)_{2}\left(\mathrm{C}_{7} \mathrm{H}_{4} \mathrm{ClO}_{2}\right)_{2}$
}

\author{
Z.-X. Li and X.-L. Zhang* \\ Baoji College of Arts and Sciences, Department of Chemistry and Chemical Engineering, Baoji, 721007 P. R. China
}

Received June 13, 2005, accepted and available on-line July 29, 2005; CCDC no. 1267/1575

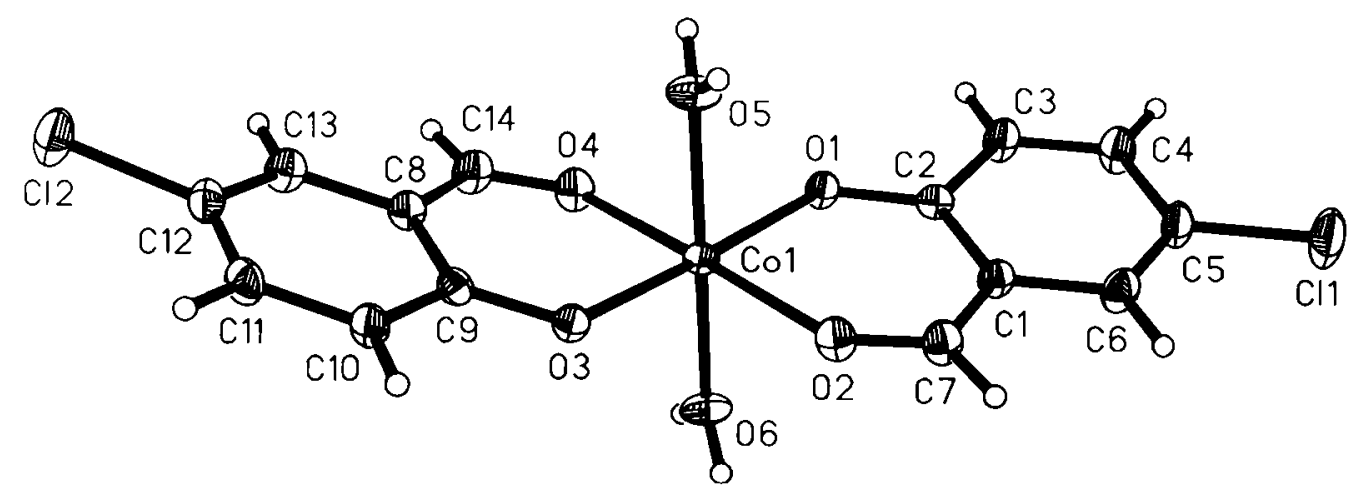

Abstract

$\mathrm{C}_{14} \mathrm{H}_{12} \mathrm{Cl}_{2} \mathrm{CoO}_{6}$, monoclinic, $P 12{ }_{1} / c 1$ (no. 14), $a=15.412(2) \AA, b=7.474(2) \AA, c=14.733(2) \AA$, $\beta=116.703(2)^{\circ}, V=1516.1 \AA^{3}, Z=4, R_{\mathrm{gt}}(F)=0.039$, $w R_{\mathrm{ref}}\left(F^{2}\right)=0.101, T=298 \mathrm{~K}$.

\section{Source of material}

5-Chlorosalicylaldehyde ( $0.1 \mathrm{mmol}, 15.7 \mathrm{mg})$ and cobalt(II) acetate tetrahydrate $(0.1 \mathrm{mmol}, 24.9 \mathrm{mg})$ were dissolved in methanol $(10 \mathrm{ml})$. The mixture was stirred for $30 \mathrm{~min}$ at room temperature to give a clear brown solution. After allowing the resulting solution to stand in air for $11 \mathrm{~d}$, brown block-shaped crystals were formed at the bottom of the vessel by slow evaporation of the solvent. The crystals were isolated by filtration, washed with methanol and dried in a vacuum desiccator using anhydrous $\mathrm{CaCl}_{2}$ (yield $54 \%$ ). Elemental analysis: found - C, $41.3 \%$; H, $3.1 \%$; calc. for $\mathrm{C}_{14} \mathrm{H}_{12} \mathrm{Cl}_{2} \mathrm{CoO}_{6}-\mathrm{C}, 41.4 \% ; \mathrm{H}, 3.0 \%$.

\section{Experimental details}

The hydrogen atoms bound to oxygen atoms were located from Fourier difference maps and refined with the $\mathrm{O}-\mathrm{H}$ and $\mathrm{H} \cdots \mathrm{H}$ distances restrained to $0.84(1) \AA$ and $1.37(2) \AA$, respectively. The other $\mathrm{H}$ atom were calculated and refined using riding rigid-body approximation.

\section{Discussion}

Cobalt complexes are of great interest in coordination chemistry in relation to catalysis and enzymatic reactions, magnetism and molecular architectures [1-2]. The complex reported here is an extension of the work on structural characterization of such cobalt complexes.

The title compound is a mononuclear cobalt(II) complex being structurally similar to the nickel(II) and zinc(II) complexes derived from other Schiff base ligands $[3,4]$. The Co(II) ion is in an octahedral coordination by four $\mathrm{O}$ atoms from two 5 -chloro-

\footnotetext{
* Correspondence author (e-mail: baojizhangxinli@yahoo.com.cn)
}

salicylaldehydato ligands defining the basal plane, and by two $O$ atoms from two coordinated water molecules occupying the axial positions. The three trans angles at $\mathrm{Col}$ are all close to $180^{\circ}$. The other angles are close to $90^{\circ}$, varying from $84.79(8)^{\circ}$ to $95.96(8)^{\circ}$, which indicates a slightly distorted octahedral geometry of the $\mathrm{Col}$ atom. The bond lengths around the $\mathrm{Col}$ atom are ranging from $2.025(2) \AA$ to $2.112(2) \AA$. The dihedral angle between the two benzene rings is $20.4(3)^{\circ}$.

Table 1. Data collection and handling.

\begin{tabular}{ll}
\hline Crystal: & $\begin{array}{l}\text { brown block, } \\
\text { size } 0.04 \times 0.09 \times 0.11 \mathrm{~mm}\end{array}$ \\
Wavelength: & Mo $K_{\alpha}$ radiation $(0.71073 \AA)$ \\
$\mu:$ & $15.12 \mathrm{~cm}^{-1}$ \\
Diffractometer, scan mode: & Siemens SMART CCD, $\omega / \varphi$ \\
$2 \theta_{\text {max }}$ & $53^{\circ}$ \\
$N(h k l)_{\text {measured, }} N(h k l)_{\text {)unique: }}:$ & 11892,3150 \\
Criterion for $I_{\text {obss }} N(h k l)_{g t}:$ & $I_{\text {obs }}>2 \sigma\left(l_{\text {obs }}\right), 2178$ \\
$N(\text { param })_{\text {refined }}$ & 224 \\
Program: & SHELXTL [5]
\end{tabular}

Table 2. Atomic coordinates and displacement parameters (in $\AA^{2}$ ).

\begin{tabular}{llllll}
\hline Atom & Site & $x$ & $y$ & $z$ & $U_{\text {iso }}$ \\
\hline H(3) & $4 e$ & 0.6835 & 0.1691 & 0.4635 & 0.046 \\
H(4) & $4 e$ & 0.8491 & 0.1752 & 0.5571 & 0.053 \\
H(6) & $4 e$ & 0.8788 & 0.3210 & 0.3130 & 0.047 \\
H(7) & $4 e$ & 0.7530 & 0.3251 & 0.1543 & 0.045 \\
H(10) & $4 e$ & 0.3203 & 0.2195 & -0.2184 & 0.041 \\
H(11) & $4 e$ & 0.1641 & 0.1181 & -0.3087 & 0.045 \\
H(13) & $4 e$ & 0.1300 & 0.1023 & -0.0569 & 0.042 \\
H(14) & $4 e$ & 0.2470 & 0.1814 & 0.0975 & 0.044 \\
H(5A) & $4 e$ & $0.474(2)$ & $-0.087(3)$ & $0.152(2)$ & $0.04(1)$ \\
H(6A) & $4 e$ & $0.530(2)$ & $0.588(3)$ & $0.102(2)$ & $0.037(9)$ \\
H(5B) & $4 e$ & $0.515(2)$ & $-0.092(3)$ & $0.088(2)$ & $0.05(1)$ \\
H(6B) & $4 e$ & $0.481(2)$ & $0.590(3)$ & $0.160(2)$ & $0.035(9)$ \\
& & & & & \\
\hline
\end{tabular}


Table 3. Atomic coordinates and displacement parameters (in $\AA^{2}$ ).

\begin{tabular}{|c|c|c|c|c|c|c|c|c|c|c|}
\hline Atom & Site & $x$ & $y$ & $z$ & $U_{11}$ & $U_{22}$ & $U_{33}$ & $U_{12}$ & $U_{13}$ & $U_{23}$ \\
\hline $\operatorname{Co}(1)$ & $4 e$ & $0.49956(3)$ & $0.25352(5)$ & $0.12423(2)$ & $0.0277(2)$ & $0.0259(2)$ & $0.0195(2)$ & $0.0002(2)$ & $0.0128(2)$ & $0.0004(2)$ \\
\hline $\mathrm{Cl}(1)$ & $4 e$ & $1.00406(6)$ & $0.2587(1)$ & $0.51670(7)$ & $0.0267(4)$ & $0.123(1)$ & $0.0518(6)$ & $-0.0057(5)$ & $0.0087(4)$ & $0.0187(6)$ \\
\hline $\mathrm{Cl}(2)$ & $4 e$ & $0.01735(6)$ & $0.0061(1)$ & $-0.25793(6)$ & $0.0317(4)$ & $0.0716(6)$ & $0.0498(5)$ & $-0.0121(4)$ & $0.0121(4)$ & $-0.0066(5)$ \\
\hline$O(1)$ & $4 e$ & $0.5805(1)$ & $0.2321(2)$ & $0.2766(1)$ & $0.025(1)$ & $0.042(1)$ & $0.021(1)$ & $-0.0024(8)$ & $0.0124(9)$ & $0.0019(8)$ \\
\hline$O(2)$ & $4 e$ & $0.6235(2)$ & $0.2720(3)$ & $0.1033(1)$ & $0.033(1)$ & $0.052(1)$ & $0.024(1)$ & $0.003(1)$ & $0.016(1)$ & $0.0035(9)$ \\
\hline$O(3)$ & $4 e$ & $0.4188(1)$ & $0.2578(2)$ & $-0.0287(1)$ & $0.028(1)$ & $0.038(1)$ & $0.021(1)$ & $-0.0031(9)$ & $0.0131(9)$ & $0.0018(8)$ \\
\hline$O(4)$ & $4 e$ & $0.3753(2)$ & $0.2454(2)$ & $0.1449(2)$ & $0.034(1)$ & $0.049(1)$ & $0.028(1)$ & $0.001(1)$ & $0.020(1)$ & $-0.002(1)$ \\
\hline$O(5)$ & $4 e$ & $0.4892(2)$ & $-0.0282(3)$ & $0.1147(2)$ & $0.060(2)$ & $0.026(1)$ & $0.044(1)$ & $0.004(1)$ & $0.037(1)$ & $0.002(1)$ \\
\hline$O(6)$ & $4 e$ & $0.5082(2)$ & $0.5277(3)$ & $0.1342(2)$ & $0.059(2)$ & $0.024(1)$ & $0.045(1)$ & $-0.001(1)$ & $0.039(1)$ & $-0.000(1)$ \\
\hline$C(1)$ & $4 e$ & $0.7375(2)$ & $0.2776(4)$ & $0.2811(2)$ & $0.028(2)$ & $0.037(2)$ & $0.028(2)$ & $0.000(1)$ & $0.016(1)$ & $0.001(1)$ \\
\hline$C(2)$ & $4 e$ & $0.6758(2)$ & $0.2359(3)$ & $0.3268(2)$ & $0.032(2)$ & $0.027(2)$ & $0.027(2)$ & $0.001(1)$ & $0.016(1)$ & $-0.001(1)$ \\
\hline$C(3)$ & $4 e$ & $0.7216(2)$ & $0.1983(4)$ & $0.4315(2)$ & $0.030(2)$ & $0.064(2)$ & $0.025(2)$ & $-0.004(2)$ & $0.016(1)$ & $0.005(2)$ \\
\hline C(4) & $4 e$ & $0.8210(2)$ & $0.2031(5)$ & $0.4882(2)$ & $0.032(2)$ & $0.074(2)$ & $0.026(2)$ & $-0.001(2)$ & $0.012(1)$ & $0.008(2)$ \\
\hline$C(5)$ & $4 e$ & $0.8790(2)$ & $0.2493(4)$ & $0.4426(2)$ & $0.022(2)$ & $0.058(2)$ & $0.037(2)$ & $-0.000(1)$ & $0.010(1)$ & $0.002(2)$ \\
\hline$C(6)$ & $4 e$ & $0.8389(2)$ & $0.2876(4)$ & $0.3422(2)$ & $0.031(2)$ & $0.057(2)$ & $0.038(2)$ & $-0.002(2)$ & $0.023(2)$ & $0.003(2)$ \\
\hline$C(7)$ & $4 e$ & $0.7052(2)$ & $0.2938(4)$ & $0.1735(2)$ & $0.032(2)$ & $0.056(2)$ & $0.031(2)$ & $0.003(2)$ & $0.019(2)$ & $0.006(2)$ \\
\hline$C(8)$ & $4 e$ & $0.2660(2)$ & $0.1803(4)$ & $-0.0300(2)$ & $0.029(2)$ & $0.035(2)$ & $0.026(2)$ & $0.000(1)$ & $0.015(1)$ & $-0.001(1)$ \\
\hline$C(9)$ & $4 e$ & $0.3265(2)$ & $0.2130(3)$ & $-0.0780(2)$ & $0.026(2)$ & $0.029(2)$ & $0.025(2)$ & $0.004(1)$ & $0.012(1)$ & $0.001(1)$ \\
\hline$C(10)$ & $4 e$ & $0.2839(2)$ & $0.1924(4)$ & $-0.1841(2)$ & $0.031(2)$ & $0.051(2)$ & $0.023(2)$ & $-0.002(2)$ & $0.013(1)$ & $0.004(1)$ \\
\hline C(11) & $4 e$ & $0.1899(2)$ & $0.1331(4)$ & $-0.2387(2)$ & $0.036(2)$ & $0.049(2)$ & $0.021(2)$ & $-0.002(2)$ & $0.007(1)$ & $0.000(1)$ \\
\hline$C(12)$ & $4 e$ & $0.1337(2)$ & $0.0959(4)$ & $-0.1894(2)$ & $0.026(2)$ & $0.041(2)$ & $0.035(2)$ & $-0.001(1)$ & $0.010(1)$ & $0.001(1)$ \\
\hline$C(13)$ & $4 e$ & $0.1695(2)$ & $0.1224(4)$ & $-0.0885(2)$ & $0.033(2)$ & $0.043(2)$ & $0.037(2)$ & $0.000(1)$ & $0.022(2)$ & $-0.002(1)$ \\
\hline C(14) & $4 e$ & $0.2950(2)$ & $0.2024(4)$ & $0.0770(2)$ & $0.033(2)$ & $0.051(2)$ & $0.036(2)$ & $-0.001(2)$ & $0.024(2)$ & $-0.001(2)$ \\
\hline
\end{tabular}

Acknowledgment. The authors thank for the research grant (no. 02js40) supported by the Phytochemistry Key Laboratory of Shaanxi province.

\section{References}

1. Fritsky, 1. O.; Oth, R.; Pritzkow, H.; Krämer, R.: Toward the redox-based allosteric control of the activity of a trinuclear metal complex catalyst. Inorg. Chim. Acta 346 (2003) 111-118.

2. Billson, T. S.; Crane, J. D.; Fox, O. D.; Heath, S. L.: A linear trinuclear mixed oxidation state cobalt(III/I//II) complex with pyrazolate bridging ligands. Inorg. Chem. Commun. 3 (2000) 718-720.
3. Stewart, J. M.; Lingafelter, E. C.; Breazeale, J. D.: The crystal structure of diaquabis(salicylaldehydato)nickel. Acta Crystallogr. 14 (1961) 888-891.

4. Erxleben, A.: Schumacher, D.: Magnesium versus zinc coordination to multidentate Schiff base ligands. Eur. J. Inorg. Chem. (2001) 3039-3046.

5. Sheldrick, G. M.: SHEL XTL. Structure Determination Software Suite. Siemens Industrial Automation, Analytical Instrumentation, USA 1995. 